\title{
Law enforcement and public health: finding common ground and global solutions to disparities in health and access to criminal justice
}

\author{
Nick Crofts* ${ }^{\star \dagger}$ and Stuart Thomas ${ }^{\ddagger}$
}

Health states are intimately related to socioeconomic status - in general, the higher the status of an individual, the better their health and the longer their life. Socioeconomic status itself relates to the major direct determinants of health, which include access to and quality of health care, the individual's social and physical environments, and their health behaviours. It also relates to indirect social determinants of health such as inequality in access to education, quality of education, income inequality, and occupational environment. At the same time, access to justice and the outcomes of people's involvement with the criminal justice system are also intimately related to socioeconomic status and social class. It is increasingly becoming recognized that these disparities affect the same communities in ways that are inextricably linked-those communities on the lower rungs of health scales also have more involvement with the criminal justice system with worse outcomes. Therefore, in order to effect change and seek to improve the health- and justicerelated outcomes for communities, both disparities must be addressed jointly.

Health inequalities which have major impacts on socially disadvantaged groups, including mental health issues, violence, and suicide, have long been acknowledged as being preventable and amenable to change-with income redistribution and equitable access to support and services (Whitehead, 1991). There is a vicious spiral in the relationship, too. Ill-health commonly worsens social disadvantage and makes it more difficult to overcome, especially among already marginalized communities; further marginalization, as happens in someone from an ethnic minority or Indigenous background who develops a mental illness, perpetuates and magnifies their marginalization even within their own communities. Professor Sir Michael Marmot, doyen of studies on social inequalities and health, has clearly demonstrated that acknowledging that the major determinants of health are social also means recognizing that the remedies for these inequalities also need to be social (Marmot, 2005).

"It is time to be explicit that the heart of a commitment to addressing health disparities is a commitment to achieving a more just society" (Braveman, et al., 2011).
People presenting frequently to health services or with health needs unmet because of lack of access to health services, and people frequently enmeshed in criminal justice services internationally are, on the whole, the same people from the same communities. They have complex issues; they are often disengaged from services, from their families and from their communities; and often present with layers of intergenerational, ingrained, and multifaceted social and economic disadvantage, with a vast array of interconnected complex needs, risks and vulnerabilities. No one agency or service is adequately equipped, resourced or even skilled enough to even begin to adequately address the multiplicity of needs presented by these individuals. This is why our current service responses and models, which remain professionally siloed, continue to fail them.

As we look towards a focus for policing for the next 50 years, in line with changing social patterns of crime and distress, and with growing understanding of their precursors and of the impacts of inequality and disparities, we must adopt more collaborative approaches. We need to fully embrace the possibilities and opportunities afforded by formally bringing together law enforcement and public health to tackle what remain highly significant and common challenges for both. It has been posited that such an approach simply leads to a 'blurring of traditional roles' (Smith, 2014) and increased workloads and conflict over territory. There may be some truth to this at times, but the boundaries reinforced by these traditional roles and mindsets, and the failure to work together to define and respect sensible shared and complementary roles, are a big part of the problem. It is disruptive thinking that facilitates progressive conversations which start with the acknowledgement of the need for a shared responsibility, then set out to determine common goals and seek innovative solutions.

Global shifts are taking place in the culture and orientation of law enforcement agencies which have seen their role as purely focused on public safety, specifically crime combat and maintenance of public order, but are increasingly beginning to understand the inextricable links between public safety and public health. In part this has resulted from deliberations about 'community policing', 'problem solving policing', 


\section{Announcing the... GLOBAL LAW ENFORCEMENT \& PUBLIC HEALTH ASSOCIATION AND ITS FORTHCOMING CONFERENCE}

The first Law Enforcement \& Public Health (LEPH) conference was held in Melbourne, Australia in 2012; its focus was on 'state of the art' thinking and collaboration. Our second conference, held in Amsterdam in 2014 (LEPH2014), moved the focus to what makes effective partnerships. Our most recent conference, again in Amsterdam, in 2016 (LEPH2016) considered the development of sustainable development goals to overcome marginalization. At this point, we created the Global Law Enforcement and Public Health Association (GLEPHA), which was legally constituted in 2017, to begin to formalize the international network of researchers, practitioners, and policy makers that has been, and continues to be, developing through these conferences. We are now delighted to announce that LEPH2018 will be held in Toronto from $21^{\text {st }}-24^{\text {th }}$ October, 2018.

The central theme of LEPH2018 will be the differential impact of socioeconomic gradients on people's health and their involvement in the criminal justice system. A particular focus of this conference will be on special interest groups and traditionally marginalized communities for whom health inequalities manifest at disproportionate levels and for whom criminal justice involvement is commonplace. Major themes at the LEPH2018 conference will include violence (especially domestic and gun-related), mental health including wellness and resilience (of first responders as well as marginalized communities), alcohol and other drugs, and corrections (prisons as public health institutions). The conference will especially examine these issues as they affect those communities hardest hit by disparities in both health and criminal justice access, including Indigenous communities and vulnerable populations.

This conference, in which the Community Safety and Knowledge Alliance is a major partner, will be an important vehicle for moving forward towards the goals of Community Safety and Wellbeing and scoping out and uncovering some innovative solutions to complex social problems. We look forward to continuing the conversation there. 'joined-up policing', and 'smart policing'. Being responsive and forward thinking has led police agencies, particularly in the Global North, to consider the range of social and economic issues that underpin insecurity and risk. Police are now engaging as nodal actors in the governance of both public safety and public health; how this is configured varies according to existing resources, skills and network actors.

CONFLICT OF INTEREST DISCLOSURES

The authors declare they have no conflicts of interest.

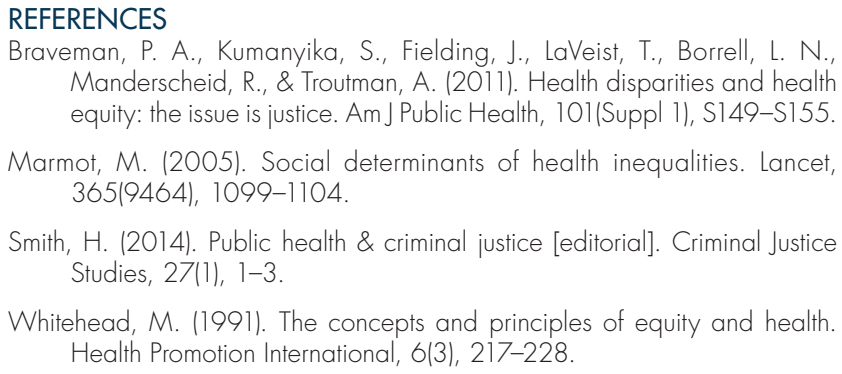

\section{AUTHOR AFFILIATIONS}

*School of Population and Global Health, University of Melbourne, Australia; ${ }^{+}$Centre for Law Enforcement and Public Health, Melbourne, Australia; ${ }^{\ddagger}$ School of Global, Urban and Social Studies, RMIT University, Melbourne, Australia. 